See discussions, stats, and author profiles for this publication at: https://www.researchgate.net/publication/320041612

\title{
Lebesgue regularity for nonlocal time-discrete equations with delays
}

Article in Fractional Calculus and Applied Analysis · July 2018

DOI: 10.1515/fca-2018-0037

CITATIONS

0

3 authors:

Claudio Leal

University of Santiago, Chile

3 PUBLICATIONS OCITATIONS

SEE PROFILE

Marina Murillo

Universitat Jaume I

33 PUBLICATIONS 103 CITATIONS

SEE PROFILE

Some of the authors of this publication are also working on these related projects:

Project Well-Posedness View project

Project Special Issue on: "Modern fractional dynamic systems and applications" View project
READS

103

Carlos Lizama

University of Santiago, Chile

159 PUBLICATIONS 1,900 CITATIONS

SEE PROFILE 


\title{
LEBESGUE REGULARITY FOR NONLOCAL TIME-DISCRETE EQUATIONS WITH DELAYS
}

\author{
Claudio Leal ${ }^{1}$, Carlos Lizama ${ }^{2}$, Marina Murillo-Arcila ${ }^{3}$
}

\begin{abstract}
In this work, we provide a new and effective characterization for the existence and uniqueness of solutions for nonlocal time-discrete equations with delays, in the setting of vector-valued Lebesgue spaces of sequences. This characterization is given solely in terms of the $R$-boundedness of the data of the problem, and in the context of the class of $U M D$ Banach spaces.

MSC 2010: Primary 34A08; Secondary 35R11, 39A14, 65Q10, 39A06.

Key Words and Phrases: fractional differences, Lebesgue regularity, time-discrete equations, delay.
\end{abstract}

\section{Introduction}

Recent technological innovations have caused a considerable interest in the study of dynamical processes that are of a mixed continuous and discrete nature. For instance, discrete-time linear models appear in the study of the solution to optimal control problems in dynamic programming [10]. Moreover, they are also used for modeling coal liquefaction mechanisms [34] and robust energy filtering in signal processing [25], among others fields of interest. In the biological context, qualitative behavior of discrete models with delays has been examined in [19] and [42. A classical textbook is the monograph by W. J. Rugh 32 .

Starting with the works of L. Weis [38], and H. Amann [6], characterizations of Lebesgue regularity using multiplier theorems for operator valued symbols have appeared in several papers in the last decade. See for instance the ones of $\mathrm{Bu}$ [12, 13, Chill and Srivastava [15, the special volume [11] and references therein.

(C) Year Diogenes Co., Sofia

pp. $\mathrm{xxx}-\mathrm{xxx}, \mathrm{DOI}$ : 
Lebesgue regularity of discrete time evolution equations in abstract spaces was first considered by S. Blünk [9] and P. Portal [30, 31]. In [20] Kovács, Li and Lubich studied maximal regularity using Blunk's results for numerical schemes. In the same line, Kemmochi [22] introduced the notion of discrete maximal regularity for the finite difference method. Other recent contributions are [4, 24 and 23]. A recent textbook on this topic is the monograph [3], where several applications in different contexts are given.

The analysis of $\ell_{p}$-maximal regularity for difference equations of fractional order $\alpha>0$ in the form

$$
\begin{cases}\Delta^{\alpha} u(n) & =T u(n)+f(n), \quad n \in \mathbb{N}_{0}, \\ u(0) & =0\end{cases}
$$

where $T$ is a bounded operator defined on a Banach space $X$ was studied in [26] for the range $0<\alpha \leq 1$ and in [27] for $1<\alpha \leq 2$. Here $\Delta^{\alpha}$ denotes the fractional difference operator of order $\alpha>0$ in the RiemannLiouville sense, see Definition 2.2 below. In [28] $\ell_{p}$-maximal regularity for the equation (1.1) with infinite delay was studied in $\mathbb{Z}$ for all $\alpha>0$ when $T$ is an unbounded operator. Recently, in [29] the authors characterized the $\ell_{p}$-maximal regularity for the finite delayed equation

$$
\begin{cases}\Delta^{\alpha} u(n) & =T u(n)+\beta u(n-\tau)+f(n), \quad n \in \mathbb{N}_{0}, \quad n \geq 1, \quad \beta \in \mathbb{R} \\ u(j) & =0, \quad j=-\tau, \ldots, 0, \quad \tau \in \mathbb{N}_{0}\end{cases}
$$

whenever $0<\alpha \leq 1$. However, the validity of such characterization for the case of $1<\alpha \leq 2$ was left as an open problem.

The main purpose of this work is to give a positive answer to this open problem.

An interesting feature that involves our model is that the fractional difference operator $\Delta^{\alpha}$ can be realized as sampling, by means of the Poisson distribution, of the classical fractional Riemann-Liouville operator. See [26, Theorem 3.5] where this remarkable connection has been discovered. This nonlocal operator has recently appeared in several research of increasing interest to different but related fields. For instance, in relation to the notion of Césaro operators of order $\alpha>0$ [2], chaos for fractional delayed logistic maps [39] and almost automorphic solutions of fractional difference equations [1]. Concerning applications, we note that fractional difference models have been considered in areas such as nano-mechanics [35, 36], economics [37, numerics [40, 41] and stability [14] among others.

In order to present our main results, this paper is organized as follows: In Section 2 we provide the reader information about differences of fractional order and we show the main methods on operator-valued Fourier 
multipliers that we will use. In Section 3, we introduce the new concept of $\alpha^{\tau}$ resolvent operators in the range $1<\alpha \leq 2$, which is an important tool for the construction of the solution of (1.1). This family, denoted by $\left\{M_{\alpha}(n)\right\}_{n \geq-\tau}$, incorporates directly the finite delay in its definition. Then, we will prove that a general solution for our model, with initial conditions $u(j)=x_{j}, j=-\tau, \ldots, 0,1$, can be written as

$$
\begin{aligned}
u(n) & =M_{\alpha}(n) u(0)+F_{\alpha}(n-1)[u(1)-u(0)] \\
& +\beta \sum_{j=1}^{\tau} F_{\alpha}(n-2+j-\tau) u(-j)+\left(F_{\alpha} * f\right)(n-2), n \geq 2 .
\end{aligned}
$$

Here, $h_{\alpha}(n)=(\alpha-1)^{n}$ and $F_{\alpha}(n)=\left(M_{\alpha} * h_{\alpha}\right)(n)$. Note that in the case $\alpha=2$ and $\beta=0$, the resolvent family $M_{2}(n)$ perfectly coincides with the notion of discrete cosine operator which was introduced and studied by Chojnacki [16] in the context of $U M D$ Banach spaces.

We remark that the representation $(1.2)$ is not straightforward but it is one of the main tasks that we have overcome in order to achieve the solution of our problem.

Finally, in Section 4, we prove the main result of this work. We will show that if $X$ is a $U M D$ space and the condition $\sup _{n \in \mathbb{N}}\left\|M_{\alpha}(n)\right\|<\infty$ is satisfied, then the maximal $\ell_{p}$-regularity of equation (1.1) and the $R$ boundedness of the sets

$$
\left\{z^{2-\alpha}(z-1)^{\alpha}\left(z^{2-\alpha}(z-1)^{\alpha}-\beta z^{-\tau}-T\right)^{-1}:|z|=1, z \neq 1\right\},
$$

and

$$
\left\{z^{-\tau}\left(z^{2-\alpha}(z-1)^{\alpha}-\beta z^{-\tau}-T\right)^{-1}:|z|=1, z \neq 1\right\},
$$

are equivalent. This characterization coincides perfectly as the counterpart of the result achieved in the paper [29] where also an $R$-boundedness condition on two sets is needed. We note that in practice, tools to check this condition are generally not easy to find. However, the monograph [3] shows a way in the general case. For the case of Hilbert spaces, we observe that $R$-boundedness can be replaced merely by uniform boundedness. For such a case, we are able to provide a very simple criterion on $T$ that ensures maximal $\ell_{p}$-regularity of equation (1.1), namely:

$\|T\|<\omega_{\alpha, \beta, \tau}:=\min _{|z|=1}\left|f_{\alpha, \beta, \tau}(z)\right|<1$ where $f_{\alpha, \beta, \tau}(z):=z^{2-\alpha}(z-1)^{\alpha}-\beta z^{-\tau}$.

See Corollary 5.1 below. We finish our work with the following examples:

$$
x(n+3)-2 x(n+2)+q x(n+1)+r x(n)=f(n),
$$

with initial conditions: $x(0)=x(1)=x(2)=0$. We show that maximal $\ell_{p^{-}}$ regularity of this equation for $f \in \ell_{p}\left(\mathbb{N}_{0}\right)$ is guaranteed whenever $1<q<2$ and $1-q<r<-1+\sqrt{2-q}$. 
In the fractional case $1<\alpha \leq 2$, we consider

$$
\Delta^{\alpha} x(n)=(1-q) x(n)-r x(n-1)+f(n)
$$

and a sufficient condition for maximal $\ell_{p^{-}}$regularity on the parameters $r, q \in \mathbb{R}$ is provided: $\omega(r):=\min _{|z|=1}\left|z^{3-\alpha}(z-1)^{\alpha}+r\right|<1$ and $1-\omega(r)<$ $q<1+\omega(r)$.

\section{Preliminaries}

In this section we define some preliminary concepts related to fractional differences, $U M D$ spaces, Fourier multiplier theorems, discrete Fourier transforms, and $R$-boundedness. In what follows we denote by $s\left(\mathbb{N}_{0} ; X\right)$ the vector space of all vector-valued sequences $f: \mathbb{N}_{0} \rightarrow X$. The forward Euler operator is defined as

$$
\Delta f(n)=f(n+1)-f(n), \quad n \in \mathbb{N}_{0},
$$

and $\Delta^{0} \equiv I$, where $I$ is the identity operator. The following fractional sum was introduced in [26, Formula 2.2]. This definition corresponds to a particular case of fractional sum proposed by Eloe and Atici in [8].

Definition 2.1. Let $\alpha>0$ and $f \in s\left(\mathbb{N}_{0} ; X\right)$ be given. We define the fractional sum of order $\alpha$ as follows

$$
\Delta^{-\alpha} f(n)=\sum_{k=0}^{n} k^{\alpha}(n-k) f(k), \quad n \in \mathbb{N}_{0},
$$

where

$$
k^{\alpha}(j)=\frac{\Gamma(\alpha+j)}{\Gamma(\alpha) \Gamma(j+1)}, \quad j \in \mathbb{N}_{0} .
$$

We also define $k^{\alpha}(j)=0$ otherwise.

REMARK 2.1. Note that $k^{\alpha}(n)$ is the $n$-th coefficient of the generating function $(1-z)^{-\alpha}$, that is,

$$
\sum_{n=0}^{\infty} k^{\alpha}(n) z^{n}=\frac{1}{(1-z)^{\alpha}}, \quad|z|<1,
$$

and therefore the kernel $\left(k^{\alpha}(n)\right)_{n \in \mathbb{N}_{0}}$ satisfies the semigroup property, $k^{\alpha} *$ $k^{\beta}=k^{\alpha+\beta}$ for $\alpha, \beta \in \mathbb{C}$.

As a function of $n, k^{\alpha}$ is increasing for $\alpha>1$, decreasing for $0<\alpha<1$ and $k^{1}(n)=1$ for $n \in \mathbb{N}$ ([43, Theorem III.1.17]). Furthermore, $k^{\alpha}(n) \leq$ $k^{\beta}(n)$ for $\beta \geq \alpha>0$ and $n \in \mathbb{N}_{0}$. 
The following definition corresponds to an analogous version of fractional derivative in the sense of Riemann-Liouville, see [7].

Definition 2.2. Let $f \in s\left(\mathbb{N}_{0} ; X\right)$ be given, we define the fractional difference operator of order $\alpha>0$ (in sense of Riemann-Liouville) as follows

$$
\Delta^{\alpha} f(n):=\Delta^{m} \circ \Delta^{-(m-\alpha)} f(n), \quad n \in \mathbb{N}_{0},
$$

where $m-1<\alpha<m, m=\lceil\alpha\rceil$.

Recall that the finite convolution between two sequences $f, g \in s\left(\mathbb{N}_{0} ; X\right)$ is defined by:

$$
(f * g)(n):=\sum_{j=0}^{n} f(n-j) g(j), \quad n \in \mathbb{N}_{0} .
$$

The $Z$-transform of a sequence $f \in s\left(\mathbb{N}_{0} ; X\right)$ is defined by

$$
\tilde{f}(z):=\sum_{j=0}^{\infty} z^{-j} f(j),
$$

where $z$ is a complex number. Note that this series is convergent for $|z|>R$, for a sufficiently large $R$. The discrete time Fourier transform of a sequence $f \in s(\mathbb{Z} ; X)$ is defined by

$$
\widehat{f}(z):=\sum_{j \in \mathbb{Z}} z^{-j} f(j), \quad \text { where } z=e^{i t}, t \in(-\pi, \pi),
$$

whenever the right side of the above identity exists. The inverse transform is given by

$$
\check{f}(n)=\frac{1}{2 \pi i} \int_{C} f(z) z^{n-1} d z=\text { sum of residues of } f(z) z^{n-1},
$$

where $C$ is a circle centered at the origin of the complex plane, that encloses all poles of $u(z) z^{n-1}$.

We finish this section with the following Fourier multiplier theorem established by S. Blunck 9 in the context of $U M D$ Banach spaces (for more information, see [5, Section III.4.3-III.4.5]). Firstly, we recall the notion of $R$-boundedness.

Definition 2.3. Let $X$ and $Y$ be two Banach spaces. A subset of $\mathcal{T} \subset \mathcal{B}(X, Y)$ is said to be $R$-bounded if there exists a constant $C \geq 0$ such that

$$
\left\|\left(T_{1} x_{1}, \ldots, T_{n} x_{n}\right)\right\|_{R} \leq C\left\|\left(x_{1}, \ldots, x_{n}\right)\right\|_{R},
$$


for each $T_{1}, \ldots, T_{n} \in \mathcal{T}, x_{1}, \ldots, x_{n} \in X, n \in \mathbb{N}$, where

$$
\left\|\left(x_{1}, \ldots, x_{n}\right)\right\|_{R}:=\frac{1}{2^{n}} \sum_{\varepsilon_{j} \in\{-1,1\}^{n}}\left\|\sum_{j=1}^{n} \varepsilon_{j} x_{j}\right\|,
$$

for $x_{1}, \ldots, x_{n} \in X$.

REMARK 2.2. Note that the Banach space $\mathcal{B}(X, Y)$ is equipped with the uniform operator topology.

For more details about $R$-boundedness and its properties see $[3$, Section $2.2]$. In what follows we denote by $\mathbb{T}:=(\pi, \pi) \backslash\{0\}$.

Theorem 2.1. [9, Theorem 1.3] Let $p \in(1, \infty)$ and let $X$ be a UMD space. Let $M: \mathbb{T} \longrightarrow \mathcal{B}(X)$ be differentiable function such that the set

$$
\left\{M(t),(z-1)(z+1) M^{\prime}(t): z=e^{i t}, t \in \mathbb{T}\right\}
$$

is $R$-bounded. Then there exists an operator $T_{M} \in \mathcal{B}\left(\ell_{p}(\mathbb{Z} ; X)\right)$ such that

$$
\left(\widehat{T_{M} f}\right)(z)=M(t) \widehat{f}(z), \quad \text { for all } \quad z=e^{i t}, t \in \mathbb{T} .
$$

The converse of Blunck's Theorem also holds without any restriction on the Banach space $X$.

Theorem 2.2. [9, Proposition 1.3] Let $p \in(1, \infty)$ and let $X$ be a $U M D$ space. Let $M: \mathbb{T} \longrightarrow \mathcal{B}(X)$ be an operator-valued function. Suppose that there exists an operator $T_{M} \in \mathcal{B}\left(\ell_{p}(\mathbb{Z} ; X)\right)$ such that the identity (2.7) holds. Then the set

$$
\{M(t): t \in \mathbb{T}\}
$$

is $R$-bounded.

\section{Resolvent families with delay: $1<\alpha \leq 2$}

In this section we study the existence and uniqueness of solutions for the following problem.

$$
\left\{\begin{array}{l}
\Delta^{\alpha} u(n)=T u(n)+\beta u(n-\tau)+f(n), \quad n \in \mathbb{N}_{0}, \tau \in \mathbb{N}_{0}, \beta \in \mathbb{R} \\
u(j)=x_{j}, j=-\tau, \ldots, 0,1,
\end{array}\right.
$$

where $1<\alpha \leq 2$ and $T \in \mathcal{B}(X)$. We start with the following definition.

Definition 3.1. Let $T$ be a bounded linear operator defined in a Banach space $X$, and let $1<\alpha \leq 2$ and $\tau \in \mathbb{N}$ be given. We say that 
$T$ is a generator of an $\alpha^{\tau}$-resolvent sequence if there exists a sequence of bounded and linear operators $\left\{M_{\alpha}(n)\right\}_{n \geq-\tau} \subset \mathcal{B}(X)$ that satisfies the following properties

(i) $M_{\alpha}(0)=M_{\alpha}(1)=I$,

(ii) $M_{\alpha}(-j)=0, j=1, \ldots, \tau$,

(iii) $\left.\Delta M_{\alpha}(n+1)\right)=T\left(M_{\alpha} * k^{\alpha-1}\right)(n)+\Delta k^{\alpha-1}(n+1) I-(2-\alpha) k^{\alpha-1}(n+$ 1) $I+\beta\left(M_{\alpha}^{\tau} * k^{\alpha-1}\right)(n)$ for each $n \in \mathbb{N}_{0}$,

where $\left\{M_{\alpha}^{\tau}(n)\right\}_{n \in \mathbb{N}_{0}}$ is defined by $M_{\alpha}^{\tau}(n):=M_{\alpha}(n-\tau)$.

REmark 3.1. Note that in the case when $\beta=0$, Definition 3.1 coincides with the definition of resolvent sequence defined in [27].

ExAmple 3.1. Suppose that $\left\{z^{2-\alpha}(z-1)^{\alpha}-\beta z^{-\tau}\right\}_{z \in C} \subset \rho(T)$, where $\rho(T)$ denotes the resolvent set of $T$ and $C$ is a circle centered at the origin that encloses all singularities of $z^{n}(z-(\alpha-1))\left(z^{2-\alpha}(z-1)^{\alpha}-\beta z^{-\tau}\right)^{-1}$ in its interior. Then for any $n \in \mathbb{N}, n \geq 2$, the formula

$$
M_{\alpha}(n):= \begin{cases}\frac{1}{2 \pi i} \int_{C} & z^{n}(z-(\alpha-1))\left(z^{2-\alpha}(z-1)^{\alpha}-\beta z^{-\tau}-T\right)^{-1} d z \\ I & n=0,1 \\ 0 & n=-\tau, \ldots,-1,\end{cases}
$$

defines an $\alpha^{\tau}$-resolvent sequence of operators with generator $T$. This fact can be formally checked using the time discrete Fourier transform method to equation (3.8) and comparing it with the formula given in Theorem 3.1 below.

Now, we recall the following Lemma proved in [29].

Lemma 3.1. Let $1<\alpha \leq 2, a: \mathbb{N}_{0} \longrightarrow \mathbb{C}$ and $S: \mathbb{N}_{0} \longrightarrow X$ be given. Then

$\Delta^{\alpha}(a * S)(n)=\sum_{j=0}^{n} \Delta^{\alpha} S(n-j) a(j)+S(0) a(n+2)-\alpha S(0) a(n+1)+S(1) a(n+1)$.

Before establishing the main result of this section, we define for any $1<\alpha \leq 2$ the sequences

$$
h_{\alpha}(n):= \begin{cases}(\alpha-1)^{n} & n \in \mathbb{N}_{0} \\ 0 & \text { otherwise }\end{cases}
$$


and

$$
F_{\alpha}(n):= \begin{cases}\left(M_{\alpha} * h_{\alpha}\right)(n) & n \in \mathbb{N}_{0} \\ 0 & \text { otherwise }\end{cases}
$$

Theorem 3.1. Let $1<\alpha \leq 2$ and $f \in s\left(\mathbb{N}_{0} ; X\right)$ be given. Assume that $T$ is a generator of an $\alpha^{\tau}$-resolvent sequence $M_{\alpha}(n)$. Then the unique solution of (3.8) is given by

$$
\begin{aligned}
u(n+2) & =M_{\alpha}(n+2) u(0)+\left(M_{\alpha} * h_{\alpha}\right)(n+1)[u(1)-u(0)] \\
& +\beta \sum_{j=1}^{\tau} F_{\alpha}(n-\tau+j) u(-j)+\left(M_{\alpha} * h_{\alpha} * f\right)(n), \quad n \in \mathbb{N}_{0} .
\end{aligned}
$$

P r o o f. We define a vector-valued sequence $v$ as follows. For $n=$ $-\tau, \ldots, 0,1, v(n)=x_{n}$ and for $n \geq 2$,

$v(n)=M_{\alpha}(n) u(0)+\left(M_{\alpha} * h_{\alpha}\right)(n-1)[u(1)-u(0)]+\beta \sum_{j=1}^{\tau} F_{\alpha}(n-2-\tau+j) u(-j)$.

First, we will show that $v$ is a solution of $(3.8)$ with $f=0$. Indeed, applying $\Delta^{\alpha}$ to $v$, we get

$$
\begin{aligned}
\Delta^{\alpha} v(n+2) & =\Delta^{\alpha} M_{\alpha}(n+2) u(0)+\Delta^{\alpha}\left(M_{\alpha} * h_{\alpha}\right)(n+1)[u(1)-u(0)] \\
& +\beta \sum_{j=1}^{\tau} \Delta^{\alpha} F_{\alpha}(n-\tau+j) u(-j), n \in \mathbb{N}_{0} .
\end{aligned}
$$

From the Definition 3.1, we have that

$$
\begin{aligned}
\Delta^{\alpha} M_{\alpha}(n+2) & =\Delta^{\alpha} M_{\alpha}(n+1)+T \Delta^{\alpha} F_{\alpha}(n)+\Delta^{\alpha} k^{\alpha-1}(n+2) I \\
& -(\alpha-1) \Delta^{\alpha} k^{\alpha-1}(n+1) I+\beta \Delta^{\alpha}\left(M_{\alpha}^{\tau} * k^{\alpha-1}\right)(n),
\end{aligned}
$$

for all $n \in \mathbb{N}_{0}$. Since $\Delta^{\alpha} k^{\alpha-1} \equiv 0$, then for any $n \in \mathbb{N}_{0}$ we have

$\Delta^{\alpha} M_{\alpha}(n+2)=\Delta^{\alpha} M_{\alpha}(n+1)+T \Delta^{\alpha}\left(M_{\alpha} * k^{\alpha-1}\right)(n)+\beta \Delta^{\alpha}\left(M_{\alpha}^{\tau} * k^{\alpha-1}\right)(n)$,

From Lemma 3.1, we obtain that

$$
\begin{aligned}
\Delta^{\alpha}\left(M_{\alpha} * k^{\alpha-1}\right)(n) & =\left(\Delta^{\alpha} k^{\alpha-1} * M_{\alpha}\right)(n)+M_{\alpha}(n+2) \\
& -\alpha M_{\alpha}(n+1)+(\alpha-1) M_{\alpha}(n+1) \\
& =M_{\alpha}(n+2)-M_{\alpha}(n+1)=\Delta M_{\alpha}(n+1),
\end{aligned}
$$

Thus, replacing (3.12) in (3.11), we obtain for any $n \in \mathbb{N}_{0}$

$$
\Delta^{\alpha} M_{\alpha}(n+2)=\Delta^{\alpha} M_{\alpha}(n+1)+T \Delta M_{\alpha}(n+1)+\beta \Delta M_{\alpha}^{\tau}(n+1),
$$


or equivalently

$$
\Delta \Delta^{\alpha} M_{\alpha}(n+1)=T \Delta M_{\alpha}(n+1)+\beta \Delta M_{\alpha}^{\tau}(n+1) .
$$

From (3.13), if $\Delta^{\alpha} M_{\alpha}(0)=T M_{\alpha}(0)+\beta M_{\alpha}(-\tau)=T$ we get $\Delta^{\alpha} M_{\alpha}(n+$ $1)=T M_{\alpha}(n+1)+\beta M_{\alpha}^{\tau}(n+1)$ for all $n \in \mathbb{N}_{0}$. Indeed, from Definition 2.3 and definition of $k^{\alpha}$, we have

$$
\begin{aligned}
\Delta^{\alpha} M_{\alpha}(n) & =\Delta^{2}\left(k^{2-\alpha} * M_{\alpha}\right)(n)=\left(k^{2-\alpha} * M_{\alpha}\right)(n+2) \\
& -2\left(k^{2-\alpha} * M_{\alpha}\right)(n+1)+\left(k^{2-\alpha} * M_{\alpha}\right)(n) .
\end{aligned}
$$

In particular, for $n=0$

$$
\Delta^{\alpha} M_{\alpha}(0)=\left(k^{2-\alpha} * M_{\alpha}\right)(2)-2\left(k^{2-\alpha} * M_{\alpha}\right)(1)+\left(k^{2-\alpha} * M_{\alpha}\right)(0) .
$$

Since

$$
\begin{aligned}
\left(k^{2-\alpha} * M_{\alpha}\right)(2) & =k^{2-\alpha}(0) M_{\alpha}(2)+k^{2-\alpha}(1) M_{\alpha}(1)+k^{2-\alpha}(2) M_{\alpha}(0) \\
& =T+(5-2 \alpha) I
\end{aligned}
$$

and

$$
\left(k^{2-\alpha} * M_{\alpha}\right)(1)=k^{2-\alpha}(1) M_{\alpha}(0)+k^{2-\alpha}(0) M_{\alpha}(1)=(3-\alpha) I
$$

as well as

$$
\left(k^{2-\alpha} * M_{\alpha}\right)(0)=k^{2-\alpha}(0) M_{\alpha}(0)=I .
$$

Replacing (3.17), (3.16) and (3.15) in (3.14), we get that

$$
\Delta^{\alpha} M_{\alpha}(0)=T+(5-2 \alpha) I-2(3-\alpha) I+I=T .
$$

On the other hand $T M_{\alpha}(0)+\beta M_{\alpha}^{\tau}(0)=T$. By Lemma 3.1 .

$$
\begin{aligned}
\Delta^{\alpha}\left(M_{\alpha} * h_{\alpha}\right)(n) & =\left(\Delta^{\alpha} M_{\alpha} * h_{\alpha}\right)(n)+h_{\alpha}(n+2)-(\alpha-1) h_{\alpha}(n+1) \\
& =\left(\Delta^{\alpha} M_{\alpha} * h_{\alpha}\right)(n) \\
& =T\left(M_{\alpha} * h_{\alpha}\right)(n)+\beta\left(M_{\alpha}^{\tau} * h_{\alpha}\right)(n) .
\end{aligned}
$$

Finally, we conclude that

$$
\begin{aligned}
\Delta^{\alpha} v(n) & =T M_{\alpha}(n) u(0)+\beta M_{\alpha}^{\tau}(n) u(0)+T\left(M_{\alpha} * h_{\alpha}\right)(n-1)[u(1)-u(0)] \\
& +\beta\left(M_{\alpha}^{\tau} * h_{\alpha}\right)(n-1)[u(1)-u(0)]+\beta \sum_{j=1}^{\tau} T F_{\alpha}(n-2-\tau+j) u(-j) \\
& +\beta^{2} \sum_{j=1}^{\tau}\left(F_{\alpha}^{\tau} * h_{\alpha}\right)(n-2-\tau+j) u(-j)=T v(n)+\beta v(n-\tau) .
\end{aligned}
$$


Then, (3.10) is the solution of the equation (3.8) with $f=0$. Now, we define a vector-valued sequence $w$ as follows

$$
w(n)= \begin{cases}\left(M_{\alpha} * h_{\alpha} * f\right)(n-2), & n \geq 2, \\ 0 & n=-\tau, \ldots, 1 .\end{cases}
$$

Since $M_{\alpha}(n)=0$, for all $n=-\tau, \ldots,-1$, from Lemma 3.1 and the last claim, we obtain

$$
\begin{aligned}
\Delta^{\alpha} w(n) & =\Delta^{\alpha}\left(M_{\alpha} * h_{\alpha} * f\right)(n-2) \\
& =\left(\Delta^{\alpha}\left(M_{\alpha} * h_{\alpha}\right) * f\right)(n-2)+\left(M_{\alpha} * h_{\alpha}\right)(0) f(n) \\
& -\alpha\left(M_{\alpha} * h_{\alpha}\right)(0) f(n-1)+\left(M_{\alpha} * h_{\alpha}\right)(1) f(n-1) \\
& =T\left(M_{\alpha} * h_{\alpha} * f\right)(n-2)+\beta\left(M_{\alpha}^{\tau} * h_{\alpha} * f\right)(n-2)+f(n) \\
& =T w(n)+\beta w(n-\tau)+f(n),
\end{aligned}
$$

for all $n \geq 2$. Then, $w$ solves (3.8) with initial conditions $w(j)=0, j=$ $-\tau, \ldots, 0,1$. Finally we claim that $u=v+w$ is solution of (3.8). Indeed,

$$
\begin{aligned}
\Delta^{\alpha} u(n) & =T v(n)+\beta v(n-\tau)+T w(n)+\beta w(n-\tau)+f(n) \\
& =T u(n)+\beta u(n-\tau)+f(n), n \in \mathbb{N}_{0}
\end{aligned}
$$

and $u(j)=x_{j}$, for $j=-\tau, \ldots, 1$ and the theorem is proved.

\section{Maximal $\ell_{p}$-regularity}

Let $T \in \mathcal{B}(X)$ and $f \in s\left(\mathbb{N}_{0} ; X\right)$ be given. In this section, we consider the following nonlocal time-discrete equation with delay $\tau \in \mathbb{N}$ :

$$
\begin{cases}\Delta^{\alpha} u(n) & =T u(n)+\beta u(n-\tau)+f(n), n \in \mathbb{N}_{0}, \\ u(j) & =0, j=-\tau, \ldots, 1,\end{cases}
$$

where $1<\alpha \leq 2$ and $\beta \in \mathbb{R}$. Assume that $T$ is a generator of an $\alpha^{\tau}$ resolvent sequence $M_{\alpha}(n)$. Since $u(j)=0$ for all $j=-\tau, \ldots, 1$ we obtain by Theorem 3.1, that the solution of $(4.19)$ can be represented by

$$
u(n)=\left(M_{\alpha} * h_{\alpha} * f\right)(n-2), \quad n \in \mathbb{N}, \quad n \geq 2 .
$$

Furthermore, from Lemma 3.1, we have the representation

$$
\Delta^{\alpha} u(n)=T\left(M_{\alpha} * h_{\alpha} * f\right)(n-2)+\beta\left(M_{\alpha}^{\tau} * h_{\alpha} * f\right)(n-2)+f(n) .
$$

This motivates the following definition.

Definition 4.1. Let $1<p<\infty, 1<\alpha \leq 2$ and $T \in \mathcal{B}(X)$ be given and suppose that $T$ is a generator of an $\alpha^{\tau}$-resolvent sequence $M_{\alpha}(n)$. We 
say that the equation 4.19 has maximal $\ell_{p}$-regularity if the operators $\mathcal{K}_{\alpha}$ and $\mathcal{P}_{\alpha}$, defined by

$$
\begin{aligned}
& \left(\mathcal{K}_{\alpha} f\right)(n):=T \sum_{j=0}^{n} M_{\alpha}(n-j)\left(h_{\alpha} * f\right)(j) \text { and } \\
& \left(\mathcal{P}_{\alpha} f\right)(n):=\sum_{j=0}^{n} M_{\alpha}^{\tau}(n-j)\left(h_{\alpha} * f\right)(j),
\end{aligned}
$$

are linear bounded operators in $\ell_{p}\left(\mathbb{N}_{0} ; X\right)$ for some $p>1$.

REMARK 4.1. Observe that, in contrast with the continuous context, the discrete maximal $\ell_{p}$-regularity ensures the stability of the solution and its fractional difference in the sense that $|u(n)| \rightarrow 0$ and $\left|\Delta^{\alpha} u(n)\right| \rightarrow 0$ as $n \rightarrow \infty$.

In what follows we need the following hypothesis:

$$
\begin{aligned}
(H)_{\alpha} & \sup _{n \in \mathbb{N}_{0}}\left\|M_{\alpha}(n)\right\|<\infty, \text { and } \\
& \left(z^{2-\alpha}(z-1)^{\alpha} I-\beta z^{-\tau}-T\right) \text { is invertible, for all }|z|=1, z \neq 1 .
\end{aligned}
$$

Now, we prove the main result of this paper.

Theorem 4.1. Let $1<p<\infty, 1<\alpha \leq 2$ and let $X$ be a $U M D$ space. Let $T \in \mathcal{B}(X)$ be given such that $T$ is a generator of an $\alpha^{\tau}$-resolvent sequence $M_{\alpha}(n)$ and the hypothesis $(H)_{\alpha}$ is satisfied. Then the following assertions are equivalent.

(i) Equation 4.19 has maximal $\ell_{p}$-regularity.

(ii) The sets

$$
\begin{gathered}
\left\{z^{2-\alpha}(z-1)^{\alpha}\left(z^{2-\alpha}(z-1)^{\alpha}-\beta z^{-\tau}-T\right)^{-1}:|z|=1, z \neq 1\right\}, \\
\left\{z^{-\tau}\left(z^{2-\alpha}(z-1)^{\alpha}-\beta z^{-\tau}-T\right)^{-1}:|z|=1, z \neq 1\right\}
\end{gathered}
$$

are $R$-bounded.

P r o o f. Suppose that $(i i)$ holds. Then we define $N(t)=z^{2-\alpha}(z-$ $1)^{\alpha}\left(z^{2-\alpha}(z-1)^{\alpha}-\beta z^{-\tau}-T\right)^{-1}$ y $S(t)=z^{-\tau}\left(z^{2-\alpha}(z-1)^{\alpha}-\beta z^{-\tau}-T\right)^{-1}$ for all $z=e^{i t}, t \in(-\pi, \pi)$. Moreover, if we denote $f_{\alpha}(t)=e^{2 i t}\left(1-e^{-i t}\right)^{\alpha}$, then we can rewrite $N(t)=f_{\alpha}(t)\left(f_{\alpha}(t)-\beta e^{-i t \tau}-T\right)^{-1}$ y $S(t)=e^{-i t \tau}\left(f_{\alpha}(t)-\right.$ $\left.\beta e^{-i t \tau}-T\right)^{-1}$. Since $f_{\alpha}^{\prime}(t)=\left(2 i+\frac{i \alpha}{e^{i t}-1}\right) f_{\alpha}(t)$, a simple computation 
gives us

$$
\begin{aligned}
N^{\prime}(t) & =\left(2 i+\frac{i \alpha}{e^{i t}-1}\right)\left(N(t)-N(t)^{2}\right)-\beta i \tau N(t) S(t) \\
S^{\prime}(t) & =-i \tau S(t)-\beta i \tau S(t)^{2}-\left(2 i+\frac{i \alpha}{e^{i t}-1}\right) N(t) S(t) .
\end{aligned}
$$

Then,

$$
\begin{aligned}
(z-1)(z+1) N^{\prime}(t) & =a_{\alpha}(t) N(t)-a_{\alpha}(t) N(t)^{2}-\beta b_{\tau}(t) N(t) S(t) \\
(z-1)(z+1) S^{\prime}(t) & =-b_{\tau}(t) S(t)-\beta b_{\tau}(t) S(t)^{2}-a_{\alpha}(t) N(t) S(t),
\end{aligned}
$$

where $a_{\alpha}(t)=2 i(z-1)(z+1)+i \alpha(z+1)$ and $b_{\tau}(t)=-i \tau(z+1)(z-1)$ are clearly bounded for $z=e^{i t}, t \in(-\pi, \pi)$. We conclude from [3], Proposition 2.2.5] that the sets

$$
\begin{gathered}
\left\{(z-1)(z+1) N^{\prime}(t): z=e^{i t}, t \in \mathbb{T}_{0}\right\} \text { and } \\
\left\{(z-1)(z+1) S^{\prime}(t): z=e^{i t}, t \in \mathbb{T}_{0}\right\}
\end{gathered}
$$

are $R$-bounded. Then, by Blunck's Theorem 2.1, we conclude that there exist operators $T_{\alpha}, U_{\alpha} \in \mathcal{B}\left(\ell_{p}(\mathbb{Z} ; X)\right)$ such that

$$
\begin{aligned}
& \widehat{\left(T_{\alpha} f\right)}(z)=N(t) \hat{f}(z), z=e^{i t}, t \in \mathbb{T}, \\
& \widehat{\left(U_{\alpha} f\right)}(z)=S(t) \hat{f}(z), z=e^{i t}, t \in \mathbb{T},
\end{aligned}
$$

for all $f \in \ell_{p}(\mathbb{Z} ; X)$. From the identity

$$
\begin{aligned}
& T\left(z^{2-\alpha}(z-1)^{\alpha}-\beta z^{-\tau}-T\right)^{-1} \\
& =\left(z^{2-\alpha}(z-1)^{\alpha}-\beta z^{-\tau}\right)\left(z^{2-\alpha}(z-1)^{\alpha}-\beta z^{-\tau}-T\right)^{-1}-I,
\end{aligned}
$$

and (4.21), we obtain that the left hand side in the identity

$$
\begin{aligned}
& T\left(z^{2-\alpha}(z-1)^{\alpha}-\beta z^{-\tau}-T\right)^{-1} \hat{f}(z) \\
& =\left(z^{2-\alpha}(z-1)^{\alpha}-\beta z^{-\tau}\right)\left(z^{2-\alpha}(z-1)^{\alpha}-\beta z^{-\tau}-T\right)^{-1} \hat{f}(z)-\hat{f}(z),
\end{aligned}
$$

defines an operator $R_{\alpha} \in \mathcal{B}\left(\ell_{p}(\mathbb{Z} ; X)\right)$ given by $R_{\alpha} f(n)=T_{\alpha} f(n)-\beta U_{\alpha} f(n)-$ $f(n)$. Now, for each $f \in \ell_{p}(\mathbb{Z} ; X)$, we define the operator

$$
K_{\alpha} f(n)= \begin{cases}T\left(M_{\alpha} * h_{\alpha} * f\right)(n), & n \in \mathbb{N}_{0}, \\ 0 & \text { otherwise. }\end{cases}
$$

Observe that the $Z$-transform of $M_{\alpha} * h_{\alpha}$ exists by hypothesis $(H)_{\alpha}$ and definition of $h_{\alpha}$, and

$$
\left(z^{2-\alpha}(z-1)^{\alpha}-\beta z^{-\tau}-T\right){\widehat{M} \alpha h_{\alpha}}_{\alpha}(z)=z^{2} I .
$$


Then, from the identity (4.21), we have that the discrete Fourier transform of $K_{\alpha} f(n-2)$ coincides with the discrete Fourier transform of $R_{\alpha} f(n)$ for $n \geq 2$. So, $K_{\alpha} f(n-2)=R_{\alpha} f(n)$ for each $n \geq 2$ by uniqueness. On the other hand, we define

$$
P_{\alpha} f(n)= \begin{cases}\left(M_{\alpha}^{\tau} * h_{\alpha} * f\right)(n) & n \in \mathbb{N}_{0} \\ 0 & \text { otherwise }\end{cases}
$$

Using again the identity (4.21), we obtain that the discrete Fourier transform of $P_{\alpha} f(n-2)$ coincides with the discrete Fourier transform of $U_{\alpha} f(n)$. So, $P_{\alpha} f(n-2)=U_{\alpha} f(n)$ for each $n \geq 2$ by uniqueness. This proves $(i)$. Now, we suppose that $(i)$ is satisfied. We define the following operators

$$
C_{\alpha} f(n)= \begin{cases}\mathcal{K}_{\alpha} f(n) & n \in \mathbb{N}_{0} \\ 0 & \text { otherwise }\end{cases}
$$

and

$$
D_{\alpha} f(n)= \begin{cases}\mathcal{P}_{\alpha} f(n) & n \in \mathbb{N}_{0} \\ 0 & \text { otherwise }\end{cases}
$$

Then, $C_{\alpha}$ and $D_{\alpha}$ are bounded linear operators on $\ell_{p}(\mathbb{Z} ; X)$. Let $T_{\alpha} f(n):=$ $C_{\alpha} f(n-2)+f(n)$, y $U_{\alpha} f(n):=D_{\alpha} f(n-2), n \in \mathbb{Z}$. Given $z=e^{i t}, t \in$ $(-\pi, \pi)$, we have that

$$
\begin{aligned}
\widehat{T_{\alpha} f}(z) & =\sum_{j \in \mathbb{Z}} z^{-j} T_{\alpha} f(j)=\sum_{j=2}^{\infty} z^{-j} C_{\alpha} f(j-2)+\sum_{j \in \mathbb{Z}} z^{-j} f(j) \\
& =z^{-2} \sum_{j=0}^{\infty} z^{-j} C_{\alpha} f(j)+\sum_{j \in \mathbb{Z}} z^{-j} f(j)=z^{-2} \sum_{j=0}^{\infty} z^{-j} C_{\alpha} f(j)+\widehat{f}(z) .
\end{aligned}
$$

By hypothesis $(H)_{\alpha}$, the $Z$-transform of $M_{\alpha} * h_{\alpha}$ exists for $|z|=1, z \neq 1$. Finally, using the identity 4.21), we obtain

$$
\begin{aligned}
\widehat{T_{\alpha} f}(z) & =z^{-2} T\left(\widehat{\mathcal{M}_{\alpha} * h_{\alpha}}\right)(z) \widehat{f}(z)+\widehat{f}(z) \\
& =z^{-2} T z(z-(\alpha-1))\left(z^{2-\alpha}(z-1)^{\alpha}-\beta z^{-\tau}-T\right)^{-1} \frac{z}{z-(\alpha-1)} \widehat{f}(z) \\
& =T\left(z^{2-\alpha}(z-1)^{\alpha}-\beta z^{-\tau}-T\right)^{-1} \widehat{f}(z)+\widehat{f}(z) \\
& =\left(z^{2-\alpha}(z-1)^{\alpha}-\beta z^{-\tau}\right)\left(z^{2-\alpha}(z-1)^{\alpha}-\beta z^{-\tau}-T\right)^{-1} \widehat{f}(z) \\
& -\widehat{f}(z)+\widehat{f}(z) \\
& =(N(t)-\beta S(t)) \widehat{f}(z),
\end{aligned}
$$


where $\mathcal{M}_{\alpha}$ is defined by

$$
\mathcal{M}_{\alpha}(n)= \begin{cases}M_{\alpha}(n) & n \in \mathbb{N}_{0} \\ 0 & \text { otherwise }\end{cases}
$$

On the other hand,

$$
\begin{aligned}
\widehat{U_{\alpha} f}(z) & =z^{-2} \sum_{j=0}^{\infty} z^{-j} \mathcal{P}_{\alpha}(j) f(j) \\
& =z^{-2}\left(\widehat{\mathcal{M}_{\alpha}^{\tau} * h} \alpha\right)(z) \widehat{f}(z)=z^{-2} z^{-\tau}\left(\widehat{\mathcal{M}_{\alpha} * h}\right) \widehat{f}(z) \\
& =z^{-2-\tau} z(z-(\alpha-1))\left(z^{2-\alpha}(z-1)^{\alpha}-\beta z^{-\tau}-T\right)^{-1} \frac{z}{z-(\alpha-1)} \widehat{f}(z) \\
& =z^{-\tau}\left(z^{2-\alpha}(z-1)^{\alpha}-\beta z^{-\tau}-T\right)^{-1} \widehat{f}(z)=S(t) \widehat{f}(z),
\end{aligned}
$$

where $\mathcal{M}_{\alpha}^{\tau}$ is defined by $\mathcal{M}_{\alpha}^{\tau}(n)=\mathcal{M}_{\alpha}(n-\tau)$.

Then, from Theorem 2.2, we conclude that $(i i)$ holds.

REMARK 4.2. In the case of Hilbert spaces, $R$-boundedness coincides with boundedness. See e.g. 3. As a consequence, condition (ii) of Theorem 4.1 can be replaced by the following equivalent assertion:

$$
\begin{gathered}
\sup _{|z|=1, z \neq 1}\left\|z^{2-\alpha}(z-1)^{\alpha}\left(z^{2-\alpha}(z-1)^{\alpha}-\beta z^{-\tau}-T\right)^{-1}\right\|<\infty \text { and } \\
\sup _{|z|=1, z \neq 1}\left\|z^{-\tau}\left(z^{2-\alpha}(z-1)^{\alpha}-\beta z^{-\tau}-T\right)^{-1}\right\|<\infty .
\end{gathered}
$$

REMARK 4.3. With the same proof and obvious modifications, the theorem is also true when we consider a finite number of delays in the equation 4.19.

We immediately obtain the following corollary (compare [29]).

Corollary 4.1. If the hypothesis of Theorem 4.1 hold, then we have $u, \Delta^{\alpha} u, T u \in \ell_{p}\left(\mathbb{N}_{0} ; X\right)$ and there exists a constant $C>0$ (independent of $\left.f \in \ell_{p}\left(\mathbb{N}_{0} ; X\right)\right)$ such that the following inequality holds

$$
\left\|\Delta^{\alpha} u\right\|_{\ell_{p}\left(\mathbb{N}_{0} ; X\right)}+\|u\|_{\ell_{p}\left(\mathbb{N}_{0} ; X\right)}+\|T u\|_{\ell_{p}\left(\mathbb{N}_{0} ; X\right)} \leq C\|f\|_{\ell_{p}\left(\mathbb{N}_{0} ; X\right)} .
$$

\section{Applications}

Let us consider the following difference equation

$$
x(n+3)-2 x(n+2)+q x(n+1)+r x(n)=f(n),
$$


where $q, r \in \mathbb{R}$. This equation was studied in the homogeneous case by Györi and Ladas in [21] and in [18, Section 5.1]. We study a particular case of this equation with initial conditions $x(0)=x(1)=x(2)=0$. Note that this equation can be reformulated as follows

$$
\Delta^{2} x(n)=(1-q) x(n)-r x(n-1)+f(n-1),
$$

with initial conditions $x(-1)=x(0)=x(1)=0$. Note that equation (5.24) can be posed into the scheme of (4.19) with $\alpha=2, T=(1-q) I, \beta=-r$ and $\tau=1$. We first compute the family $\left\{M_{\alpha}(n)\right\}_{n \geq-1}$ in order to obtain a solution $x$ of (5.24). Indeed, using the inverse formula of Z-transform, we get that

$$
\begin{aligned}
M_{2}(n) & =\frac{1}{2 \pi i} \int_{C} z^{n-1} z(z-1)\left((z-1)^{2}+r z^{-1}-(1-q)\right)^{-1} d z \\
& =\frac{1}{2 \pi i} \int_{C} \frac{z^{n+1}(z-1)}{\left(z^{3}-2 z^{2}+q z+r\right)} d z \\
& =\frac{1}{2 \pi i} \int_{C} \frac{z^{n+1}(z-1)}{\left(z-\lambda_{1}\right)\left(z-\lambda_{2}\right)\left(z-\lambda_{3}\right)} d z \\
& =\frac{\left(\lambda_{1}^{n+2}-\lambda_{1}^{n+1}\right)}{\left(\lambda_{1}-\lambda_{2}\right)\left(\lambda_{1}-\lambda_{3}\right)}-\frac{\left(\lambda_{2}^{n+2}-\lambda_{2}^{n+1}\right)}{\left(\lambda_{1}-\lambda_{2}\right)\left(\lambda_{2}-\lambda_{3}\right)}+\frac{\left(\lambda_{3}^{n+2}-\lambda_{3}^{n+1}\right)}{\left(\lambda_{1}-\lambda_{3}\right)\left(\lambda_{2}-\lambda_{3}\right)},
\end{aligned}
$$

where $C$ is a circle centered at the origin that encloses the roots $\lambda_{1}, \lambda_{2}, \lambda_{3}$ of the equation $z^{3}-2 z^{2}+q z+r=0$ in its interior.

It follows from Schur-Cohn criterion (see [18, Theorem 5.1]) or Samuelson criterion (see for example [33]) that all these roots lie inside of the unitary disc $\mathbb{D}$ if and only if $|r-2|<1+q$ and $|q+2 r|<1-r^{2}$ which, in turn, is equivalent to $1<q<2$ and $1-q<r<-1+\sqrt{2-q}$. See Figure 1 below. Then, under this restriction on the parameters of equation (5.24), we obtain that $\sup _{n \in \mathbb{N}}\left|M_{2}(n)\right|<\infty$. That means that the first part of the condition $(H)_{2}$ hold. In particular, we also have $z^{3}-2 z^{2}+q z+r \neq 0$ for $|z|=1$ and consequently

$$
\sup _{|z|=1, z \neq 1}\left|(z-1)^{2}\left((z-1)^{2}+r z^{-1}-(1-q)\right)^{-1}\right|<\infty
$$

and

$$
\sup _{|z|=1, z \neq 1}\left|z^{-1}\left((z-1)^{2}+r z^{-1}-(1-q)\right)^{-1}\right|<\infty .
$$

Therefore all the conditions given in Theorem 4.1 holds and we conclude that whenever $1<q<2$ and $1-q<r<-1+\sqrt{2-q}$ and $f \in \ell_{p}\left(\mathbb{N}_{0}\right)$, there exists a unique $u \in \ell_{p}\left(\mathbb{N}_{0}\right)$ solving (5.24). 


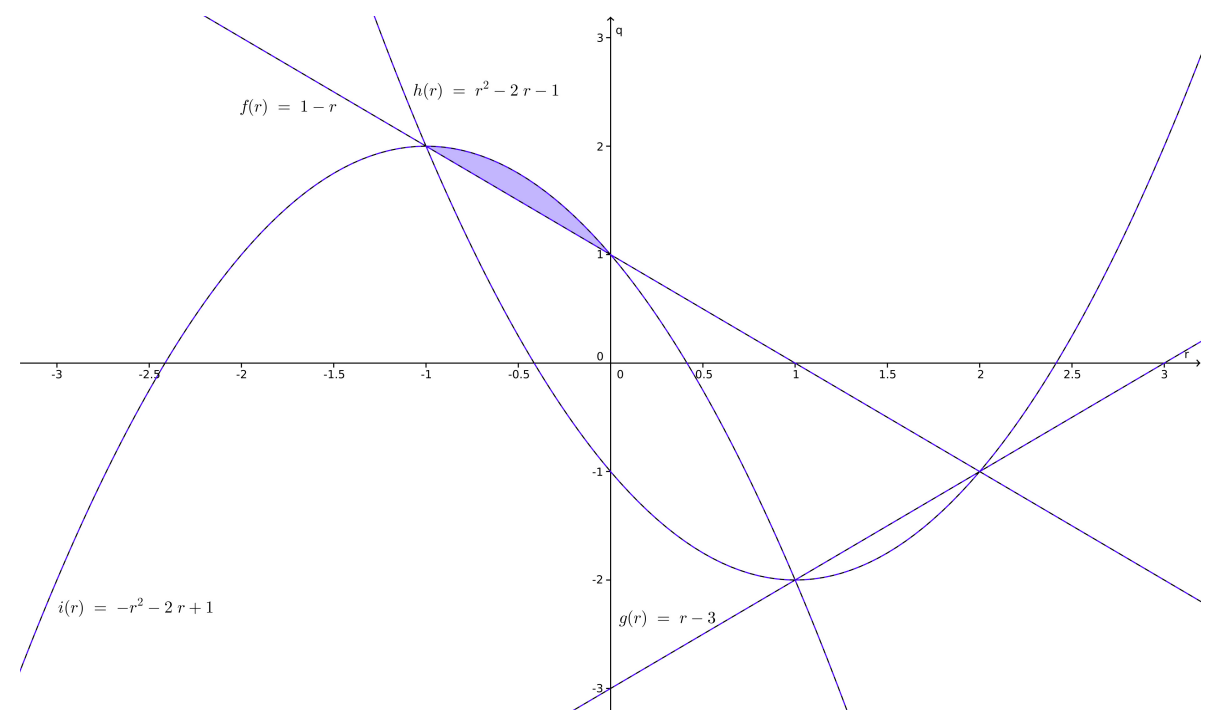

FiguRE 1. The sector $|r-2|<1+q$ and $|q+2 r|<1-r^{2}$

In order to handle fractional models, the following result will be useful.

Corollary 5.1. Let $1<\alpha \leq 2, \beta \in \mathbb{R}, \tau \in \mathbb{N}_{0}$. Let $X$ be a Hilbert space and $T \in \mathcal{B}(X)$ satisfying the following condition

$\|T\|<\omega_{\alpha, \beta, \tau}:=\min _{|z|=1}\left|f_{\alpha, \beta, \tau}(z)\right|<1$ where $f_{\alpha, \beta, \tau}(z):=z^{2-\alpha}(z-1)^{\alpha}-\beta z^{-\tau}$.

Then equation 4.19) has maximal $\ell_{p}$-regularity.

P r o o f. We first prove that $T$ is the generator of an $\alpha^{\tau}$-resolvent sequence $M_{\alpha}(n)$ and the hypothesis $(H)_{\alpha}$ is satisfied. Indeed, by hypothesis and an application of the minimum principle, we obtain that $f_{\alpha, \beta, \tau}(z) \in$ $\rho(T)$ and

$$
\left(f_{\alpha, \beta, \tau}(z)-T\right)^{-1}=\sum_{n=0}^{\infty} \frac{T^{n}}{\left(f_{\alpha, \beta, \tau}(z)\right)^{n+1}},
$$

whenever $|z| \leq 1$. Hence there exists a circle $\Gamma$ centered at the origin of radius $R<1$ such that for any $n \in \mathbb{N}, n \geq 2$,

$$
M_{\alpha}(n):=\left\{\begin{array}{l}
\frac{1}{2 \pi i} \int_{\Gamma} z^{n}(z-(\alpha-1))\left(z^{2-\alpha}(z-1)^{\alpha}-\beta z^{-\tau}-T\right)^{-1} d z, \\
I \quad n=0,1 \\
0 \quad n=-\tau, \ldots, 0
\end{array}\right.
$$


defines an $\alpha^{\tau}$-resolvent family. Observe that we also have

$$
\left\|\left(f_{\alpha, \beta, \tau}(z)-T\right)^{-1}\right\| \leq \frac{1}{\left|f_{\alpha, \beta, \tau}(z)\right|-\|T\|}<\frac{1}{\omega_{\alpha, \beta, \tau}-\|T\|}
$$

As a consequence, for all $n \in \mathbb{N}$, we have

$$
\left\|M_{\alpha}(n)\right\|<\frac{R^{n+1}(R+|\alpha-1|)}{\omega_{\alpha, \beta, \tau}-\|T\|}
$$

and then $\sup _{n \in \mathbb{N}}\left\|M_{\alpha}(n)\right\|<\infty$. This proves the claim. Moreover,

$$
\sup _{|z|=1, z \neq 1}\left\|z^{1-\alpha}(z-1)^{\alpha}\left(f_{\alpha, \beta, \tau}(z)-T\right)^{-1}\right\|<\infty
$$

and

$$
\sup _{|z|=1, z \neq 1}\left\|z^{-\tau}\left(f_{\alpha, \beta, \tau}(z)-T\right)^{-1}\right\|<\infty
$$

Then part (ii) of Theorem 4.1 holds and we conclude that equation 4.19 has maximal $\ell_{p}$-regularity.

ExAMPle 5.1. Motivated by the model given by (5.24) we consider the fractional equation

$$
\Delta^{\alpha} x(n)=(1-q) x(n)-r x(n-1)+f(n-1), \quad 1<\alpha \leq 2,
$$

with initial conditions $x(-1)=x(0)=x(1)=0$. We illustrate the validity of the condition in the previous corollary plotting the graph of the complex function

$$
f_{\alpha,-r, 1}(z)=z^{2-\alpha}(z-1)^{\alpha}+r z^{-1},|z|=1,
$$

for different values of $1<\alpha \leq 2$ and $r \in \mathbb{R}$. Observe that given $\alpha$ and $r$, there are cases where there exists a number $q$ satisfying the hypothesis of Corollary 5.1. See the graphs below. Moreover, the graphs show that $\omega_{\alpha,-r, 1} \rightarrow 0$ as $\alpha \rightarrow 1$ for some values of $r$ (for instance when $r=0.6$ ). 


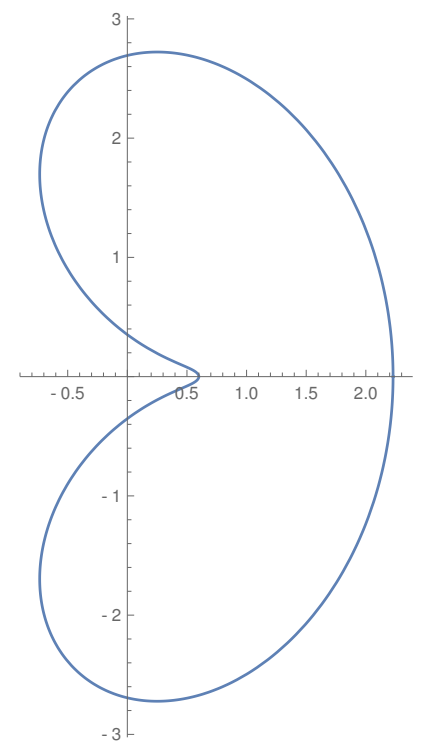

FiguRE 2. $\alpha=1.5$ and $r=0.6$. Observe that the minimum value $\omega_{1.5,-0.6,1}$ is attained approximately at 0.5 and consequently $0.5<q<1.5$.

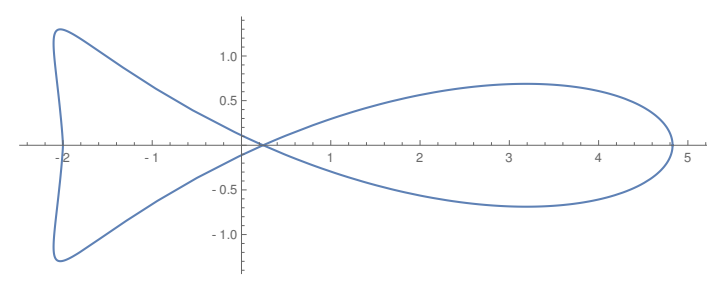

Figure 3. $\alpha=1.5$ and $r=-2$. Observe that the minimum value $\omega_{1.5,-0.6,1}$ is attained approximately at 0.2 and consequently $0.8<q<1.2$.

\section{Acknowledgements}

C. Lizama is partially supported by PIA Anillo ACT 1416.

\section{References}

[1] L. Abadias, C. Lizama, Almost automorphic mild solutions to fractional partial difference-differential equations. Appl. Analysis. 95, No 6 (2016), 1347-1369. 
[2] L. Abadias, C. Lizama, P.J. Miana, M. P. Velasco, Cesáro sums and algebra homomorphisms of bounded operators. Israel J. Math. 216, No 1 (2016), 471-505.

[3] R. P. Agarwal, C. Cuevas, C. Lizama, Regularity of Difference Equations on Banach Spaces. Springer-Verlag, Cham (2014).

[4] G. Akrivis, B. Li, C. Lubich, Combining maximal regularity and energy estimates for time discretizations of quasilinear parabolic equations. Math. Comp. 86, No 306 (2017), 1527-1552.

[5] H. Amann, Linear and Quasilinear Parabolic Problems. Monographs in Mathematics, 89, Birkhäuser, Basel (1995).

[6] H. Amann, Operator-valued Fourier multipliers, vector-valued Besov spaces, and applications. Math. Nachr. 186 (1997), 5-56.

[7] F. M. Atici, P. W. Eloe, A transform method in discrete fractional calculus. Int. J. Difference Equ. 2, No 2 (2007), 165-176.

[8] F. M. Atici, P. W. Eloe, Initial value problems in discrete fractional calculus. Proc. Amer. Math. Soc. 137, No 3 (2009), 981-989.

[9] S. Blünck, Maximal regularity of discrete and continuous time evolution equations. Studia Math. 146, No 2 (2001), 157-176.

[10] F. Borrelli, M. Baotic, A. Bemporad, M. Morari, Dynamic programming for constrained optimal control of discrete-time linear hybrid systems. Automatica 41 (2005), 1709-1721.

[11] D. Bothe, R. Denk, M. Hieber, R. Schnaubelt, G. Simonett, M. Wilke, R. Zacher, Special issue: Parabolic evolution equations, maximal regularity, and applications dedicated to Jan Prüss. J. Evol. Equ. 17, No 1 (2017), 1-15.

[12] S. Bu, Mild well-posedness of equations with fractional derivative. Math. Nachr. 285, No 2/3 (2012), 202-209.

[13] $\mathrm{S}$. $\mathrm{Bu}$, Well-posedness of fractional differential equations on vectorvauled function spaces. Integral Equations Operator Theory $\mathbf{7 1}$, No 2 (2001), 259-274.

[14] J. Cermák, I. Gyóri, L. Nechvátal, On explicit stability conditions for a linear fractional difference system. Fract. Calc. Appl. Anal. 18, No 3 (2015), 651-672.

[15] R. Chill, S. Srivastava, $L^{p}$-maximal regularity for second order Cauchy problems. Math. Z. 251, No 4 (2005), 751-781.

[16] W. Chojnacki, On operator-valued cosine sequences on UMD spaces. Studia Math. 199, No 3 (2010), 267-278.

[17] C. W. Clark, A delayed-recruitment model of population dynamics, with an application to baleen whale populations. J. Math. Biol. 3 (1976), 381-391. 
[18] S. Elaydi, An Introduction to Difference Equations. Springer, New York (2005).

[19] X. Fu, M. Li, Spatial dynamics for lattice difference equations with a shifting habitat. J. Differential Equations 259 (2015), 1957-1989.

[20] B. Kovács, B. Li, C. Lubich, A-stable time discretizations preserve maximal parabolic regularity. SIAM J. Numer. Anal. 54, No 6 (2016), 3600-3624.

[21] I. Gyóri, G. Ladas, Oscillation Theory of Delay Differential Equations. Clarendon, Oxford (1991).

[22] T. Kemmochi, Discrete maximal regularity for abstract Cauchy problems. Studia Math. 234, No 3 (2016), 241-63.

[23] D. Leykekhman, B. Vexler, Discrete maximal parabolic regularity for Galerkin finite element methods. Numer. Math. 135, No 3 (2017), 923952.

[24] B. Li, W. Sun, Maximal regularity of fully discrete finite element solutions of parabolic equations. SIAM J. Numer. Anal. 55, No 2 (2017), $521-542$.

[25] Z.-M. Li, X.-H. Chang, K. Mathiyalagan, J. Xiang, Robust energyto-peak filtering for discrete-time nonlinear systems with measurement quantization. Signal Processing 139, No 1 (2017), 1339-1351.

[26] C. Lizama, $\ell_{p}$-maximal regularity for fractional difference equations on UMD spaces. Math. Nach. 288, No 17/18 (2015), 2079-2092.

[27] C. Lizama, M. Murillo-Arcila, $\ell_{p}$-maximal regularity for a class of fractional difference equations on UMD spaces: The case $1<\alpha \leq 2$. $B a$ nach J. Math. Anal. 11, No 1 (2017), 188-206.

[28] C. Lizama, M. Murillo-Arcila, Maximal regularity in $\ell_{p}$ spaces for discrete time fractional shifted equations. J. Differential Equations 263, No 6 (2017), 3175-3196.

[29] C. Lizama, M. Murillo-Arcila, Well posedness for semidiscrete abstract fractional Cauchy problems with finite delay. J. Comput. Appl. Math. 339 (2018), 356-366.

[30] P. Portal, Discrete time analytic semigroups and the geometry of Banach spaces. Semigroup Forum 67, No 1 (2003), 125-144.

[31] P. Portal, Maximal regularity of evolution equations on discrete time scales. J. Math. Anal. Appl. 304, No 1 (2005), 1-12.

[32] W. J. Rugh, Linear System Theory, 2nd edn. Prentice Hall, New York (1996).

[33] P. A. Samuelson, Conditions that the roots of a polynomial be less than unity in absolute value. Ann. Math. Stat. 12, No 3 (1941), 360-364. 
[34] E. H. Simsek, E. Gulec, H. Kavustu, Application of Kalman filter to determination of coal liquefaction mechanisms using discrete time models. Fuel 207, No 1 (2017), 814-820.

[35] V. E. Tarasov, Fractional-order difference equations for physical lattices and some applications. J. Math. Phys. 56, No 10 (2015), 1-19.

[36] V. E. Tarasov, Fractional Liouville equation on lattice phase-space. Phys. A 421 (2015), 330-342.

[37] V. V. Tarasova, V. E. Tarasov, Logistic map with memory from economic model. Chaos Solitons Fractals 95 (2017), 84-91.

[38] L. Weis, Operator-valued Fourier multiplier theorems and maximal $L_{p}$-regularity. Math. Ann. 319 (2001), 735-758.

[39] G. C. Wu, D. Baleanu, Discrete chaos in fractional delayed logistic maps. Nonlinear Dynamics 80, No 4 (2016), 1697-1703.

[40] G. C. Wu, D. Baleanu, H-P. Xie, Riesz Riemann-Liouville difference on discrete domains. Chaos 26 , No 8 (2016), 084308, 5 pp.

[41] G. C. Wu, D. Baleanu, Z. G. Deng, S. D. Zeng, Lattice fractional diffusion equation in terms of a Riesz-Caputo difference. Phys. A 438 (2015), 335-339.

[42] Z. X. Yu, Uniqueness of critical travelling waves for nonlocal lattice equations with delays. Proc. Amer. Math. Soc. 140, No 11 (2012), 3853-3859.

[43] A. Zygmund, Trigonometric Series. 2nd ed. Vols. I, II, Cambridge University Press, New York, (1959).

1 Departamento de Matemática y Ciencia de la Computación, Universidad de Santiago de Chile

Las Sophoras 173, Estación Central

Santiago, CHILE

e-mail: claudio.leal@usach.cl

Received: November 1, 2014

2 Departamento de Matemática y Ciencia de la Computación, Universidad de Santiago de Chile

Las Sophoras 173, Estación Central

Santiago, CHILE

e-mail: carlos.lizama@usach.cl

${ }^{3}$ Institut de Màtematiques i Aplicacions de Castelló (IMAC), Universitat Jaume I,

Campus del Riu s/n, 12701 Castelló, SPAIN

e-mail: murillom@uji.es 